\title{
Particles Make Their Own Movie
}

\author{
Active particles organize into mesmerizing patterns in response to a \\ time-varying electric field.
}

\section{By David Ehrenstein}

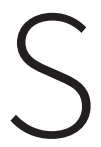
elf-propelled particles can organize themselves into structures that could eventually be put to use for advanced technologies. Many of these particles move continuously, but now researchers have shown that particles that are induced to start and stop can produce a surprising square lattice pattern that forms and reforms in a continuous

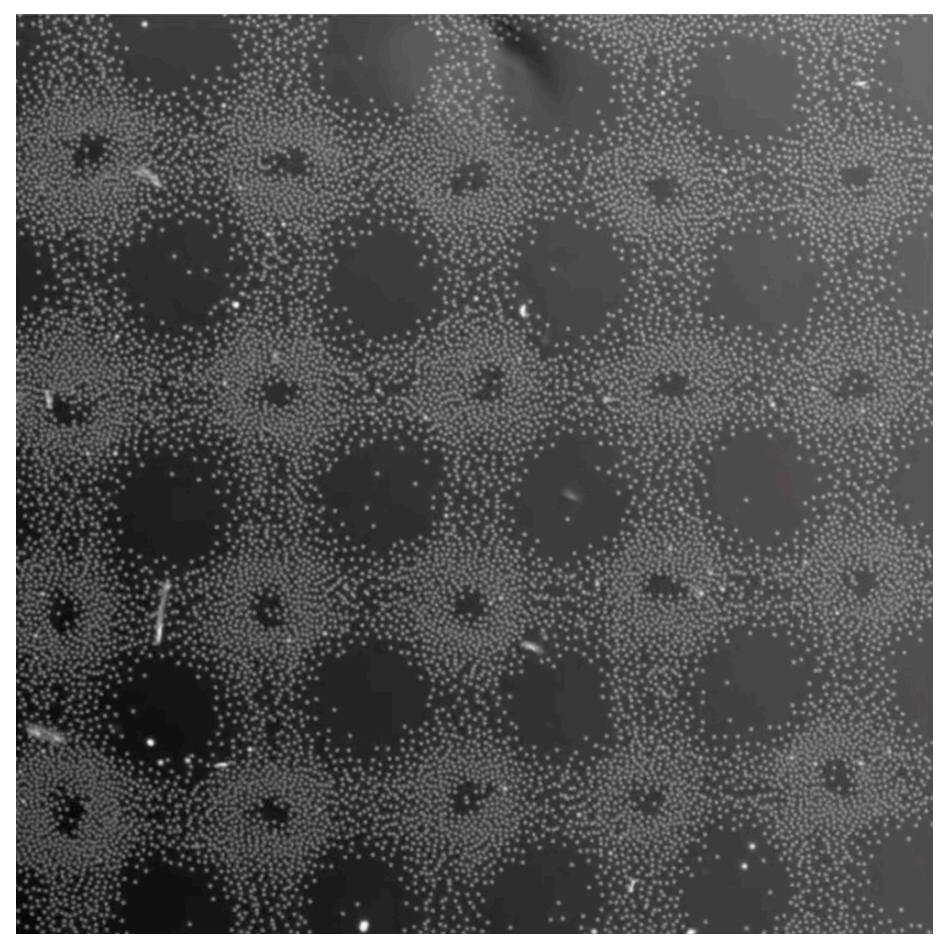

Plastic spheres in a conductive fluid form a square lattice that periodically forms, dissolves, and re-forms. Here, a 3-volt-per-micrometer electric field is applied for 112.5 milliseconds and then turned off for 12.5 milliseconds in each cycle. The video is shown at $12 \%$ of its true speed.

Credit: B. Zhang et al. [1] cycle [1]. The team believes that the technique could be applied to other types of active particles, potentially leading to a new category of active matter structures.

Alexey Snezhko and his colleagues at the Argonne National Laboratory in Illinois study a well-known system of self-propelled particles called Quincke rollers. Their rollers are 5-micrometer-diameter plastic spheres submerged in an organic conducting fluid. An external electric field perpendicular to the layer of particles causes each one to roll at a constant speed in a randomly chosen direction, thanks to an interaction of the field with surface charges on the particle. But when the density of particles is high enough, they can interact through fluid and electrostatic forces to move collectively, forming vortices or other large-scale patterns.

Snezhko and his colleagues found that periodically turning the field off for, say, 20 out of every 100 milliseconds could produce a new pattern. The particles form a square lattice of clumps, pause while the field is off, and then move out of their clumps to form a new lattice that is offset from the first one. This cycle repeats with each on-off cycle of the field.

By varying the on and off durations and other parameters and by performing simulations, the team was able to explain the phenomenon. The field leads to a repulsion between particles, so that each time the field turns on, a particle chooses the direction that would most effectively take it away from its neighbors. Initially, the cycling of the field drives the particles into randomly distributed clusters, but eventually these clusters arrange into alternating square lattice patterns.

"Temporal control of the activity represents an additional 'knob' to orchestrate emergent collective behavior and self-organization in active systems," Snezhko says. 


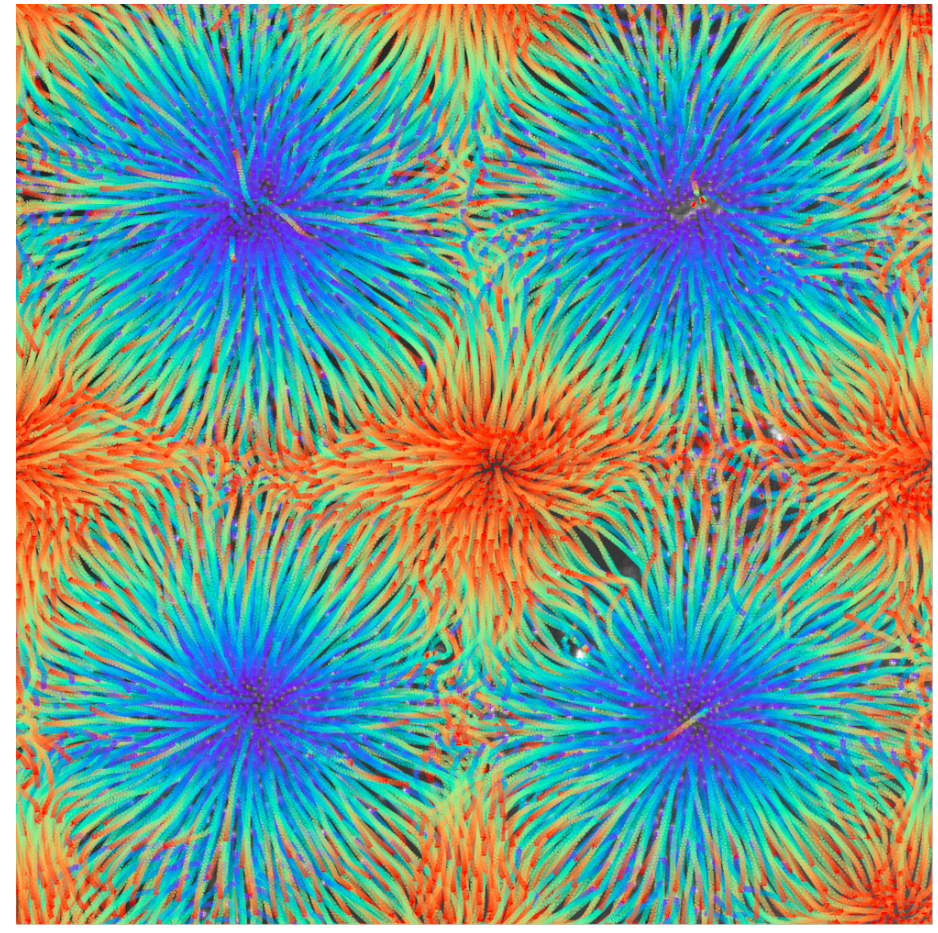

Self-organized dance. This image superimposes a sequence of many snapshots over a single cycle of the electric field, with each image color coded to indicate its time in the cycle, progressing in time from dark blue to red.

Credit: B. Zhang et al. [1]
David Ehrenstein is a Senior Editor for Physics.

\section{REFERENCES}

1. B. Zhang et al., "Guiding self-assembly of active colloids by temporal modulation of activity," Phys. Rev. Lett. 128, 018004

(2022). 